\title{
Superconvergence Analysis and Error Expansion for the Wilson Nonconforming Finite Element
}

\author{
Hongsen Chen* $\quad$ Bo $\mathrm{Li}^{\dagger}$
}

\begin{abstract}
In this paper the Wilson nonconforming finite element is considered for solving a class of two-dimensional second-order elliptic boundary value problems. Superconvergence estimates and error expansions are obtained for both uniform and non-uniform rectangular meshes. A new lower bound of the error shows that the usual error estimates are optimal. Finally a discussion on the error behaviour in negative norms shows that there is generally no improvement in the order by going to weaker norms.
\end{abstract}

Keywords: Wilson finite element, rectangular mesh, optimal error estimate, negative norm, superconvergence, error expansion, extrapolation

Subject classification: $\operatorname{AMS}(\mathrm{MOS}): 65 \mathrm{~N} 30$

\section{Introduction}

The Wilson finite element, known as Wilson's brick in three-dimensional finite element applications, is widely used in computational mechanics and structural engineering, see, e.g., [5] and [17]. The corresponding finite element space consists of piececwise

*This work was supported by the National Natural Science Foundation of P. R. China and Deutsche Forschungsgemeinschaft, SFB 359, Germany.

${ }^{\dagger}$ This work is part of the Transitions and Defects in Ordered Materials Project and was supported in part by the NSF through grant DMS 911-1572, by the AFOSR through grant AFOSR-91-0301, by the ARO through grants DAAL03-89-G-0081 and DAAL03-92-G-0003, and by a grant from the Minnesota Supercomputer Institute. 
quadratic functions which are required to be continuous only at the vertices of elements. This reduced continuity property determines the nonconforming character of the Wilson element in solving second-order elliptic boundary value problems.

The nonconforming Wilson element passes the Irons patch test on general quasiuniform quadrilateral meshes and the convergence in the energy norm is of first order, [5], [8], [13], [18]. It is shown by an example in [14] that this rate of convergence is optimal. Thus, in contrast to a conforming quadratic finite element which achieves a second-order rate of convergence, the Wilson element looses one order of accuracy due to its nonconformity. However, pointwise superconvergence has been observed in practical computations, [17]. This interesting phenomenon motivated this study of error estimates of higher order for the Wilson element.

For conforming finite elements superconvergence estimates and error expansions are well studied in the literature. We refer to [7] for a survey on various results of superconvergence and to [2] for a fundamental work on asymptotic error expansions. Some of the technical details can be found in the monograph [18]. The theory of superconvergence has reached a fairly complete stage for the conforming finite elements, though the counterexamples presented in [9] still give rise to some questions. However, in the case of nonconforming finite elements, due to the reduced continuity of trial and test functions, it becomes much more difficult to establish superconvergence properties and related asymptotic error expansions. The latter are the basis for the application of Richardson extrapolation or defect correction techniques by which the accuracy of the scheme can be largely improved. See [1] for a description of this difficulty in detail.

There seems to be no results yet in the literature on higher order error estimates based on superconvergence properties for nonconforming finite elements. For the relatively simple Wilson element, a result of superconvergence in the energy norm has been obtained in [16] for a model situation. Independently, the second author considered the Wilson element within the same setting and obtained $L^{p}$ and $W^{1, p}(1 \leq$ $p \leq \infty)$ error estimates as well as the pointwise superconvergence and extrapolation results. All these results indicate that the Wilson element is almost equivalent to the conforming bilinear finite element in terms of the asymptotic error behaviour. For a detailed description of this result, see the monograph [18].

In this paper a new approach to the analysis of superconvergence of the Wilson element is developed which now applies also to certain non-uniform meshes and to more general equations. By this method pointwise superconvergence estimates and asymptotic error expansions are derived. Furthermore, an interesting lower bound for the error is obtained which strengthens the result in [14] about the optimality of the standard error estimates for the Wilson element. The key point of our analysis is the separation of the conforming part of the nonconforming approximation and the 
use of known results for conforming finite elements, see, e.g., [2], [7] and [18]. Our analysis can directly be carried over to three-dimensional problems. We expect that our method will also work for other nonconforming finite elements, especially for those often used in solving forth-order elliptic boundary value problems, [5].

For simplicity, let $\Omega$ be the unit square in the $x y$-plane. We consider the following boundary value problem:

$$
\left\{\begin{aligned}
L u \equiv-\frac{\partial}{\partial x}\left(A_{1} \frac{\partial u}{\partial x}\right)-\frac{\partial}{\partial y}\left(A_{2} \frac{\partial u}{\partial y}\right)+B u=f, & \text { in } \quad \Omega, \\
u=0, & \text { on } \quad \partial \Omega,
\end{aligned}\right.
$$

where $A_{1}, A_{2}, B$ and $f$ are sufficiently smooth functions defined on $\Omega$, and $A_{1}, A_{2} \geq$ $\alpha=$ const. $>0, B \geq 0$. As usual, for an integer $k \geq 0$ and a real number $p$, with $1 \leq p \leq \infty, W^{k, p}(\Omega), W_{0}^{k, p}(\Omega)$ are the Sobolev spaces over the domain $\Omega$, and $\|\cdot\|_{k, p, \Omega}$, $|\cdot|_{k, p, \Omega}$ the corresponding norms and seminorms. The inner product and the norm of $L^{2}(\Omega)$ are denoted by $(\cdot, \cdot)$ and $\|\cdot\|$, respectively. The symbol $c$ is used as a generic constant varying with the context and is always assumed to be independent of both the mesh size $h$ and the solution $u$, except the dependance is otherwise indicated.

Let $T_{h}=\left\{e_{i j}\right\}_{i, j=1}^{n, m}$ be a rectangular partition of the domain $\Omega$, where $n, m$ are two positive integers, $e_{i j}=\left[x_{i-1}, x_{i}\right] \times\left[y_{j-1}, y_{j}\right]$ are rectangular elements, and

$$
0=x_{0}<x_{1}<\cdots<x_{n}=1, \quad 0=y_{0}<y_{1}<\cdots<y_{m}=1
$$

are two one-dimensional partitions on the $x$-axis and $y$-axis, respectively. Define $h_{i}=x_{i}-x_{i-1}, k_{j}=y_{j}-y_{j-1}$, and the mesh size $h=\max \left\{h_{i}, k_{j}\right\}_{i, j=1}^{n, m}$. As usual $T_{h}$ is said to be quasi-uniform if there exists a constant $c$ such that

$$
c h \leq \min \left\{h_{i}, k_{j}\right\}_{i, j=1}^{n, m} .
$$

Furthermore, $T_{h}$ is said to be unidirectionally uniform if

$$
h_{i}=h_{1}, i=1, \cdots n, \quad \text { and } \quad k_{j}=k_{1}, j=1, \cdots, m .
$$

Throughout this paper we assume that $T_{h}$ is quasi-uniform, unless any more restrictive conditions on $T_{h}$ are mentioned.

The analysis in this paper relies on the property of the differential operator $L$ to be separable with respect to the coordinate directions $x$ and $y$ and a corresponding tensor product structure of the finite element mesh. Therefore, it does not directly apply to problems involving mixed derivative terms on such a cartesian mesh. But the treatment of first order derivative terms is possible. 
Now let $N_{h}$ and $Z_{h}$ denote the set of vertices and the set of the centers of all rectangular elements in $T_{h}$, respectively. For the mesh $T_{h}$, we define $V_{h}$ to be the Wilson element space which consists of all functions $v_{h} \in L^{2}(\Omega)$ such that $v_{h}$ is piecewise quadratic over $\Omega$ and continuous on $N_{h}$, and $v_{h}$ vanishes on $N_{h} \cap \partial \Omega$. The Wilson finite element solution of the equation (1), $u_{h} \in V_{h}$, is defined through the relation

$$
a_{h}\left(u_{h}, \varphi_{h}\right)=\left(f, \varphi_{h}\right), \quad \text { for all } \varphi_{h} \in V_{h},
$$

where

$$
a_{h}\left(u_{h}, \varphi_{h}\right)=\sum_{e \in T_{h}} \int_{e}\left(A_{1} \frac{\partial u_{h}}{\partial x} \frac{\partial \varphi_{h}}{\partial x}+A_{2} \frac{\partial u_{h}}{\partial y} \frac{\partial \varphi_{h}}{\partial y}+B u_{h} \varphi_{h}\right) d x d y
$$

For later use we introduce as an auxiliary tool the following conforming bilinear finite element space $\bar{V}_{h}$ defined by

$$
\bar{V}_{h}=\left\{\bar{v}_{h} \in C(\bar{\Omega}) \cap W_{0}^{1,2}(\Omega): \bar{v}_{h} \text { is piecewise bilinear }\right\} .
$$

Notice that $\bar{V}_{h}$ is a subspace of $V_{h}$. The bilinear finite element solution of the equation (1), $\bar{u}_{h} \in \bar{V}_{h}$, is defined by

$$
a\left(\bar{u}_{h}, \bar{\varphi}_{h}\right)=\left(f, \bar{\varphi}_{h}\right), \quad \text { for all } \quad \bar{\varphi}_{h} \in \bar{V}_{h},
$$

where

$$
a\left(\bar{u}_{h}, \bar{\varphi}_{h}\right)=\int_{\Omega}\left(A_{1} \frac{\partial \bar{u}_{h}}{\partial x} \frac{\partial \bar{\varphi}_{h}}{\partial x}+A_{2} \frac{\partial \bar{u}_{h}}{\partial y} \frac{\partial \bar{\varphi}_{h}}{\partial y}+B \bar{u}_{h} \bar{\varphi}_{h}\right) d x d y
$$

The usual (conforming) Lagrange interpolation operator

$$
I_{h}: C(\bar{\Omega}) \cap W_{0}^{1,2}(\Omega) \rightarrow \bar{V}_{h}
$$

is defined by the relation

$$
\left.I_{h} \varphi\right|_{N_{h}}=\left.\varphi\right|_{N_{h}}
$$

Notice that the operator $I_{h}$ can be applied to those functions which are continuous on $N_{h}$ but may not be continuous globally. It can also be restricted to each element of $T_{h}$ in the usual way. Finally, let us introduce the notation $\|\cdot\|_{k, p, h}$ which is defined by

$$
\|\cdot\|_{k, p, h}=\left\{\begin{array}{lc}
\left(\sum_{e \in T_{h}}\|\cdot\|_{k, p, e}^{p}\right)^{1 / p}, & 1 \leq p<\infty, \\
\max _{e \in T_{h}}\|\cdot\|_{k, \infty, e}, & p=\infty .
\end{array}\right.
$$

Our main results are collected in the following theorems. 
Theorem 1 Let $u$ and $u_{h}$ be the solutions of problems (1) and (2), respectively. Suppose $u \in W^{3, \infty}(\Omega)$ and $u \not \equiv 0$. Then, there exists a constant $c_{0}>0$, depending on $u$, such that, for sufficiently small $h$,

$$
c_{0} h^{2-k} \leq\left\|u-u_{h}\right\|_{k, p, h}
$$

where $0 \leq k \leq 2$, and $1 \leq p \leq \infty$.

The assumption $u \in W^{3, \infty}(\Omega)$ in Theorem 1 , is no real restriction since the right hand side of (4) becomes even larger for a solution $u$ with lower regularity.

As a direct consequence of Theorem 1, we see that the usual error estimates for the Wilson element are optimal, [5], [8], [13], [14]. Moreover, we cannot even expect any higher order error estimates in negative norms. In general, for any sufficiently small $h$, there exists a function $f$, such that the corresponding solution $u$ satisfy

$$
c_{1} h^{2} \leq\left\|u-u_{h}\right\|_{-2, \Omega},
$$

where $c_{1}>0$ is a constant independent of $h$. We note that $\left\|u-u_{h}\right\|_{-1, \Omega}$ is of order of $O\left(h^{4}\right)$ for the conforming quadratic finite element. This observation, together with Theorem 1, indicates that the Wilson element is asymptotically not better than the conforming bilinear element. We will briefly outline the construction of such a particular right hand side $f$ leading to (5) at the end of this paper.

Next we have the pointwise superconvergence estimate for the gradient error.

Theorem 2 Suppose $T_{h}$ is unidirectionally uniform. Let $u$ and $u_{h}$ be the solutions of problems (1) and (2), respectively. If $u \in W^{3, \infty}(\Omega)$, then there holds the superconvergence estimate

$$
\max _{p \in Z_{h}}\left|\nabla\left(u-u_{h}\right)(p)\right| \leq c h^{2} \ln \frac{1}{h}\|u\|_{3, \infty, \Omega} .
$$

The result of the above theorem can be localized by using the usual arguments. For any subdomains $\Omega_{0} \subset \subset \Omega_{1} \subset \Omega$, if $T_{h}$ is unidirectionally uniform on $\Omega_{1}$, there holds

$$
\max _{p \in Z_{h} \cap \Omega_{0}}\left|\nabla\left(u-u_{h}\right)(p)\right| \leq c h^{2} \ln \frac{1}{h}\left(\|u\|_{3, \infty, \Omega_{1}}+\|u\|_{2, \Omega}\right) .
$$

We point out that estimate (6) sharpens the earlier result:

$$
\left(\sum_{p \in Z_{h} \cap \Omega_{0}}\left|\nabla\left(u-u_{h}\right)(p)\right|^{2}\right)^{1 / 2} \leq c h^{2}\|u\|_{3, \Omega}
$$


which was obtained in [16] for the Poisson equation on an uniform mesh.

Before stating our Theorem 3, let us introduce some more notation. Let $T_{h / 2}, T_{1, h / 2}$, $T_{2, h / 2}$ be the refined meshes corresponding to the three different types of refinement indicated in the following figures,
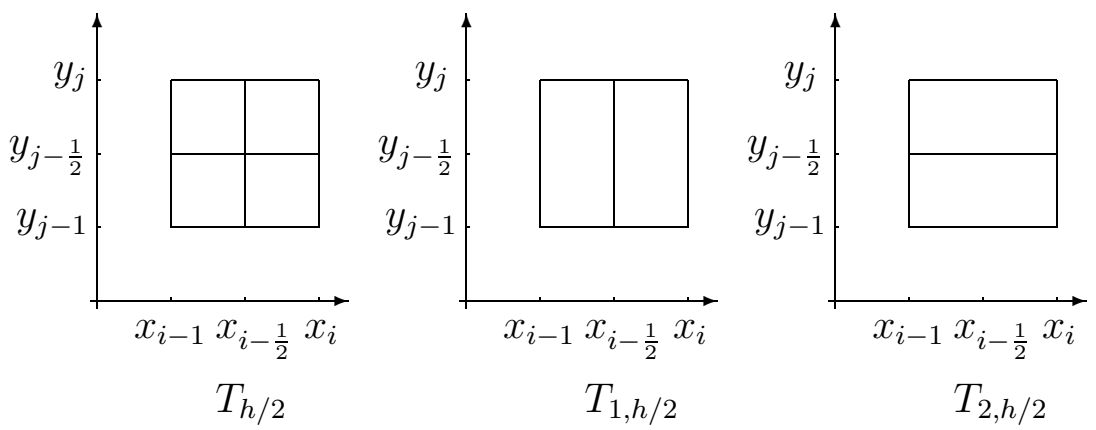

Figure 1: The refined meshes $T_{h / 2}, T_{1, h / 2}, T_{2, h / 2}$

where $x_{i-1 / 2}=\frac{x_{i-1}+x_{i}}{2}, y_{j-1 / 2}=\frac{y_{j-1}+y_{j}}{2}$. By $u_{h / 2}, u_{1, h / 2}$, and $u_{2, h / 2}$ we denote the corresponding nonconforming Wilson finite element approximations. Then we have the following result for the standard extrapolation and for the so-called unidirectional extrapolation.

Theorem 3 Let $u_{h / 2}, u_{1, h / 2}$ and $u_{2, h / 2}$ be as defined above, and let $u \in C(\bar{\Omega}) \cap W_{0}^{1,2}(\Omega)$ be the solution of (1). Further, let

$$
\gamma_{h}(q)=\left|\frac{4 u_{h / 2}(q)-u_{h}(q)}{3}-u(q)\right|+\left|\frac{4 u_{1, h / 2}(q)+4 u_{2, h / 2}(q)-5 u_{h}(q)}{3}-u(q)\right| .
$$

(i) If $u \in W^{3, \infty}(\Omega)$, and $T_{h}$ is quasi-uniform, then

$$
\max _{q \in N_{h}} \gamma_{h}(q) \leq c h^{3} \ln \frac{1}{h}\|u\|_{3, \infty, \Omega} .
$$

(ii) If $u \in W^{4, \infty}(\Omega)$, and $T_{h}$ is unidirectionally uniform, then

$$
\max _{q \in N_{h}} \gamma_{h}(q) \leq c h^{4}\left(\ln \frac{1}{h}\right)^{2}\|u\|_{4, \infty, \Omega}
$$


We remark that for any subdomain $\Omega_{0}$ uniformly bounded away from the corner points of $\partial \Omega$, there holds

$$
\max _{q \in N_{h} \cap \Omega_{0}} \gamma_{h}(q) \leq c h^{4} \ln \frac{1}{h}\|u\|_{4, \infty, \Omega},
$$

which improves on the power of the logarithm in Theorem 3 (ii).

\section{Some lemmas}

In this section we provide some technical lemmas.

Lemma 1 There exists a constant $c>0$ such that, for any $e \in T_{h}$, any $\varphi \in W^{3,1}(e)$ and any $A \in W^{2, \infty}(e)$,

$$
\begin{gathered}
\left|\int_{e} A \frac{\partial\left(\varphi-I_{h} \varphi\right)}{\partial x} d x d y\right| \leq c h^{2}\left(\|A\|_{0, \infty, e}+\left\|\frac{\partial A}{\partial x}\right\|_{0, \infty, e}+h|A|_{2, \infty, e}\right)\|\varphi\|_{3,1, e}, \\
\left|\int_{e} A \frac{\partial\left(\varphi-I_{h} \varphi\right)}{\partial y} d x d y\right| \leq c h^{2}\left(\|A\|_{0, \infty, e}+\left\|\frac{\partial A}{\partial y}\right\|_{0, \infty, e}+h|A|_{2, \infty, e}\right)\|\varphi\|_{3,1, e} .
\end{gathered}
$$

Proof. We only prove the first inequality. Let $\left(x_{0}, y_{0}\right)$ be the coordinates of the center of $e$. By Taylor's expansion formula, we have

$$
A(x, y)=A\left(x_{0}, y_{0}\right)+\frac{\partial A\left(x_{0}, y_{0}\right)}{\partial x}\left(x-x_{0}\right)+\frac{\partial A\left(x_{0}, y_{0}\right)}{\partial y}\left(y-y_{0}\right)+O\left(h^{2}\right)|A|_{2, \infty, e} .
$$

If a function $p$ is quadratic on $e$, then $\int_{e} \partial\left(p-I_{h} p\right) / \partial x d x d y=0$. Thus, by the Bramble-Hilbert Lemma, [5], together with a transformation to a reference element, it follows that

$$
\left|\int_{e} \frac{\partial\left(\varphi-I_{h} \varphi\right)}{\partial x} d x d y\right| \leq c h^{2}\|\varphi\|_{3,1, e} .
$$

Moreover, in view of standard interpolation estimates, we have

$$
\left|\int_{e}\left(x-x_{0}\right) \frac{\partial\left(\varphi-I_{h} \varphi\right)}{\partial x} d x d y\right| \leq h \int_{e}\left|\frac{\partial\left(\varphi-I_{h} \varphi\right)}{\partial x}\right| d x d y \leq c h^{2}\|\varphi\|_{3,1, e} .
$$

Combining all the above estimates, we obtain the first inequality. Q.E.D. 
Lemma 2 For any element $e \in T_{h}$, and any smooth functions $A$ and $\varphi$ defined on $e$, the following expansions hold true:

$$
\begin{gathered}
\int_{e} A\left(I_{h} \varphi-\varphi\right) d x d y=\frac{1}{12} \int_{e} A\left(h_{i}^{2} \frac{\partial^{2} \varphi}{\partial x^{2}}+k_{j}^{2} \frac{\partial^{2} \varphi}{\partial y}\right) d x d y+R^{(1)} \\
\int_{e} A \frac{\partial\left(I_{h} \varphi-\varphi\right)}{\partial x} d x d y=\frac{1}{12} \int_{e}\left(k_{j}^{2} A \frac{\partial^{3} \varphi}{\partial x \partial y^{2}}-h_{i}^{2} \frac{\partial A}{\partial x} \frac{\partial^{2} \varphi}{\partial x^{2}}\right) d x d y+R^{(2)} \\
\int_{e} A \frac{\partial\left(I_{h} \varphi-\varphi\right)}{\partial y} d x d y=\frac{1}{12} \int_{e}\left(h_{i}^{2} A \frac{\partial^{3} \varphi}{\partial y \partial x^{2}}-k_{j}^{2} \frac{\partial A}{\partial y} \frac{\partial^{2} \varphi}{\partial y^{2}}\right) d x d y+R^{(3)}
\end{gathered}
$$

where

$$
\begin{gathered}
\left|R^{(1)}\right| \leq c h^{4} \int_{e}\left(|A|\left|\partial^{4} \varphi\right|+|\partial A|\left|\partial^{3} \varphi\right|+\left|\partial^{2} A\right|\left|\partial^{2} \varphi\right|\right) d x d y \\
\left|R^{(2)}\right|+\left|R^{(3)}\right| \leq c h^{4} \int_{e}\left(|A|\left|\partial^{5} \varphi\right|+|\partial A|\left|\partial^{4} \varphi\right|+\left|\partial^{2} A\right|\left|\partial^{3} \varphi\right|+\left|\partial^{3} A\right|\left|\partial^{2} \varphi\right|\right) d x d y .
\end{gathered}
$$

Here, for a positive integer $k, \partial^{k}$ denotes a constant linear combination of all partial derivatives of order $k$, and $\partial=\partial^{1}$.

Proof. See [1], [3] or [10]. Q.E.D.

Lemma 3 For the nonconforming approximation $u_{h}$ and the conforming approximation $\bar{u}_{h}$ of problems (2) and (3), respectively, there holds the estimate

$$
\left\|I_{h} u_{h}-\bar{u}_{h}\right\|_{1, \infty, h} \leq c h^{2} \ln \frac{1}{h}\left\|u_{h}\right\|_{2, \infty, h} .
$$

Proof. Let $q \in \bar{\Omega}$ be arbitray. Define the derivative discrete Green's function $\partial g_{h}^{q} \in$ $\bar{V}_{h}$ by, [18],

$$
a\left(\varphi_{h}, \partial g_{h}^{q}\right)=\partial \varphi_{h}(q), \quad \text { for all } \varphi_{h} \in \bar{V}_{h},
$$

where $\partial=\frac{\partial}{\partial x}$ or $\partial=\frac{\partial}{\partial y}$. By (2) and (3), Lemma 1, and an inverse estimate, we have that

$$
\begin{aligned}
\partial\left(I_{h} u_{h}-\bar{u}_{h}\right)(q) & =a\left(I_{h} u_{h}-\bar{u}_{h}, \partial g_{h}^{q}\right)=a_{h}\left(I_{h} u_{h}-u_{h}, \partial g_{h}^{q}\right) \\
& \leq c h^{2} \sum_{e \in T_{h}}\left(\left\|\partial g_{h}^{q}\right\|_{1, \infty, e}+h\left\|\partial g_{h}^{q}\right\|_{2, \infty, e}\right)\left\|u_{h}\right\|_{2,1, e} \\
& \leq c h^{2} \sum_{e \in T_{h}}\left\|\partial g_{h}^{q}\right\|_{1, \infty, e}\left\|u_{h}\right\|_{2,1, e} \\
& \leq c h^{2} \sum_{e \in T_{h}}\left\|\partial g_{h}^{q}\right\|_{1,1, e}\left\|u_{h}\right\|_{2, \infty, e} \\
& \leq c h^{2}\left\|\partial g_{h}^{q}\right\|_{1,1, \Omega}\left\|u_{h}\right\|_{2, \infty, h} .
\end{aligned}
$$


Hence, by noting that (see [11] and [17])

$$
\left\|\partial g_{h}^{q}\right\|_{1,1, \Omega} \leq c \ln \frac{1}{h}
$$

we obtain

$$
\left|I_{h} u_{h}-\bar{u}_{h}\right|_{1, \infty, \Omega} \leq c h^{2} \ln \frac{1}{h}\left\|u_{h}\right\|_{2, \infty, h} .
$$

The $L^{\infty}$ estimate can be obtained similarly by applying the discrete Green's function $g_{h}^{q} \in \bar{V}_{h}$ at the point $q$, [6], [18]. Thus, the proof is complete. Q.E.D.

Lemma 4 The following relations hold for the nonconforming solution $u_{h}$ of problem (2), at any $p \in Z_{h}$,

$$
\begin{aligned}
\partial_{s}^{2} u_{h}(p)= & -\frac{1}{A_{s}(p)}\left(\partial_{s} A_{s}(p) \partial_{s} u_{h}(p)-B(p) u_{h}(p)+f(p)\right) \\
& +h^{k}\|f\|_{k, \infty, \Omega}+O\left(h^{2}\right)\left\|u_{h}\right\|_{2, \infty, h},
\end{aligned}
$$

where $s=1,2, k=0,1,2$, and $\partial_{1}^{2}=\frac{\partial^{2}}{\partial x^{2}}, \partial_{2}^{2}=\frac{\partial^{2}}{\partial y^{2}}$.

Proof. We first consider the expansion of $u_{h}$ with respect to basis functions of the space $V_{h}$. Let $q \in N_{h}$ be given and $\varphi_{q} \in \bar{V}_{h}$ be the corresponding bilinear nodal basis function. Further let $\psi_{i j}^{(1)}, \psi_{i j}^{(2)} \in V_{h}$ be defined as

$$
\begin{aligned}
& \psi_{i j}^{(1)}(x, y)=\left\{\begin{array}{lll}
\frac{\left(x-x_{0}\right)^{2}}{2}-\frac{h_{i}^{2}}{8}, & \text { on } & e_{i j}, \\
0, & \text { on } & \Omega \backslash e_{i j},
\end{array}\right. \\
& \psi_{i j}^{(2)}(x, y)=\left\{\begin{array}{lll}
\frac{\left(y-y_{0}\right)^{2}}{2}-\frac{k_{j}^{2}}{8}, & \text { on } & e_{i j}, \\
0, & \text { on } & \Omega \backslash e_{i j},
\end{array}\right.
\end{aligned}
$$

where $\left(x_{0}, y_{0}\right)$ is the center of the rectangle $e_{i j}$. All $\varphi_{q}\left(q \in N_{h}\right)$ and $\psi_{i j}^{(1)}, \psi_{i j}^{(2)}\left(e_{i j} \in T_{h}\right)$ form a basis of the Wilson finite element space $V_{h}$. The solution $u_{h}$ can thus be expressed as follows,

$$
u_{h}(x, y)=\sum_{q \in N_{h}} u_{h}(q) \varphi_{q}(x, y)+\sum_{e_{i j} \in T_{h}}\left(\frac{\partial^{2} u_{h}}{\partial x^{2}} \psi_{i j}^{(1)}+\frac{\partial^{2} u_{h}}{\partial y^{2}} \psi_{i j}^{(2)}\right) .
$$

By (2) we get

$$
\sum_{t=1}^{4} u_{h}\left(q_{t}\right) \int_{e_{i j}}\left\{A_{1} \frac{\partial \varphi_{q_{t}}}{\partial x} \frac{\partial \psi_{i j}^{(1)}}{\partial x}+B \varphi_{q_{t}} \psi_{i j}^{(1)}\right\} d x d y
$$




$$
+\int_{e_{i j}}\left\{A_{1} \frac{\partial^{2} u_{h}}{\partial x^{2}}\left(\frac{\partial \psi_{i j}^{(1)}}{\partial x}\right)^{2}+B\left(\frac{\partial^{2} u_{h}}{\partial x^{2}} \psi_{i j}^{(1)} \psi_{i j}^{(1)}+\frac{\partial^{2} u_{h}}{\partial y^{2}} \psi_{i j}^{(1)} \psi_{i j}^{(2)}\right)\right\} d x d y=\left(f, \psi_{i j}^{(1)}\right)
$$

where $q_{t}, t=1, \cdots, 4$, are the four vertices of a fixed element $e=e_{i j}$ ordered in the clockwise sense with $q_{1}$ the upper left vertex. Obviously we have that

$$
\int_{e_{i j}} B\left(\frac{\partial^{2} u_{h}}{\partial x^{2}} \psi_{i j}^{(1)} \psi_{i j}^{(1)}+\frac{\partial^{2} u_{h}}{\partial y^{2}} \psi_{i j}^{(1)} \psi_{i j}^{(2)}\right) d x d y=O\left(h^{6}\right)\left|u_{h}\right|_{2, \infty, e_{i j}} .
$$

Applying Taylor's expansion formula to $A_{1}$ at the center $p$ of $e_{i j}$, we get

$$
\int_{e_{i j}} A_{1} \frac{\partial^{2} u_{h}}{\partial x^{2}}\left(\frac{\partial \psi_{i j}^{(1)}}{\partial x}\right)^{2} d x d y=\frac{h_{i}^{3} k_{j}}{12} A_{1}(p) \frac{\partial^{2} u_{h}}{\partial x^{2}}+O\left(h^{6}\right)\left|u_{h}\right|_{2, \infty, e_{i j}}
$$

Furthermore, using the following indentities

$$
\begin{array}{cc}
\varphi_{q_{1}}=-\frac{\left(y-y_{j-1}\right)\left(x-x_{i}\right)}{h_{i} k_{j}}, & \varphi_{q_{2}}=\frac{\left(y-y_{j-1}\right)\left(x-x_{i-1}\right)}{h_{i} k_{j}}, \\
\varphi_{q_{3}}=-\frac{\left(y-y_{j}\right)\left(x-x_{i-1}\right)}{h_{i} k_{j}}, & \varphi_{q_{4}}=\frac{\left(y-y_{j}\right)\left(x-x_{i}\right)}{h_{i} k_{j}},
\end{array}
$$

and applying Taylor's expansion formula to the function $B$, we obtain that

$$
\int_{e_{i j}} B \varphi_{q_{t}} \psi_{i j}^{(1)} d x d y=-\frac{h_{i}^{3} k_{j}}{48} B(p)+\tau_{t}^{(1)} \frac{h_{i}^{4} k_{j}}{48} \frac{\partial B(p)}{\partial x}+\tau_{t}^{(2)} \frac{h_{i}^{3} k_{j}^{2}}{288} \frac{\partial B(p)}{\partial y}+O\left(h^{6}\right),
$$

where $t=1, \cdots, 4$, and

$$
\tau_{t}^{(1)}=\left\{\begin{array}{ll}
1 \quad \text { if } t=1, \text { or } 4 \\
-1 \quad \text { if } t=2 \text {, or } 3
\end{array}, \quad \text { and } \tau_{t}^{(2)}=\left\{\begin{array}{ll}
-1 & \text { if } t=1, \text { or } 2 \\
1 \quad \text { if } t=3, \text { or } 4
\end{array} .\right.\right.
$$

Moreover there hold the identities

$$
\begin{gathered}
u_{h}\left(q_{1}\right)+u_{h}\left(q_{2}\right)+u_{h}\left(q_{3}\right)+u_{h}\left(q_{4}\right)=4 u_{h}(p)+\frac{h_{i}^{2}}{2} \frac{\partial^{2} u_{h}}{\partial x^{2}}+\frac{k_{j}^{2}}{2} \frac{\partial^{2} u_{h}}{\partial y^{2}} \\
u_{h}\left(q_{1}\right)-u_{h}\left(q_{2}\right)-u_{h}\left(q_{3}\right)+u_{h}\left(q_{4}\right)=-2 h_{i} \frac{\partial u_{h}(p)}{\partial x}
\end{gathered}
$$

and

$$
-u_{h}\left(q_{1}\right)-u_{h}\left(q_{2}\right)+u_{h}\left(q_{3}\right)+u_{h}\left(q_{4}\right)=-2 k_{j} \frac{\partial u_{h}(p)}{\partial y}
$$


Thus we can conclude that

$$
\begin{aligned}
\sum_{t=1}^{4} u_{h}\left(q_{t}\right) \int_{e_{i j}} B \varphi_{q_{t}} \psi_{i j}^{(1)} d x d y= & -\frac{h_{i}^{3} k_{j}}{12} B(p) u_{h}(p)-\frac{h_{i}^{5} k_{j}}{240} \frac{\partial B(p)}{\partial x} \frac{\partial u_{h}(p)}{\partial x} \\
& -\frac{h_{i}^{3} k_{j}^{3}}{144} \frac{\partial B(p)}{\partial y} \frac{\partial u_{h}(p)}{\partial y}+O\left(h^{6}\right)\left|u_{h}\right|_{2, \infty, e_{i j}} \\
= & -\frac{h_{i}^{3} k_{j}}{12} B(p) u_{h}(p)+O\left(h^{6}\right)\left\|u_{h}\right\|_{2, \infty, e_{i j}} .
\end{aligned}
$$

Similarly one obtains

$$
\begin{aligned}
\int_{e_{i j}} A_{1} \frac{\partial \varphi_{q_{t}}}{\partial x} \frac{\partial \psi_{i j}^{(1)}}{\partial x} d x d y= & -\tau_{t}^{(1)} \frac{h_{i}^{2} k_{j}}{24} \frac{\partial A_{1}(p)}{\partial x}+(-1)^{t} \frac{h_{i}^{2} k_{j}^{2}}{144} \frac{\partial^{2} A_{1}(p)}{\partial x \partial y} \\
& -\tau_{t}^{(1)} \frac{h_{i}^{4} k_{j}}{960} \frac{\partial^{3} A_{1}(p)}{\partial x^{3}}-\tau_{t}^{(1)} \frac{h_{i}^{2} k_{j}^{3}}{576} \frac{\partial^{3} A_{1}(p)}{\partial x \partial y^{2}}+O\left(h^{6}\right) .
\end{aligned}
$$

Noting that

$$
-u_{h}\left(q_{1}\right)+u_{h}\left(q_{2}\right)-u_{h}\left(q_{3}\right)+u_{h}\left(q_{4}\right)=h_{i} k_{j} \frac{\partial^{2} u_{h}(p)}{\partial x \partial y},
$$

we have then

$$
\sum_{t=1}^{4} u_{h}\left(q_{t}\right) \int_{e_{i j}} A_{1} \frac{\partial \varphi_{q_{t}}}{\partial x} \frac{\partial \psi_{i j}^{(1)}}{\partial x} d x d y=\frac{h_{i}^{3} k_{j}}{12} \frac{\partial A_{1}(p)}{\partial x} \frac{\partial u_{h}(p)}{\partial x}+O\left(h^{6}\right)\left\|u_{h}\right\|_{2, \infty, e_{i j}} .
$$

Now, it follows from all the above relations that

$$
\frac{h_{i}^{3} k_{j}}{12}\left(\frac{\partial A_{1}(p)}{\partial x} \frac{\partial u_{h}(p)}{\partial x}-B(p) u_{h}(p)+A_{1}(p) \frac{\partial^{2} u_{h}(p)}{\partial x^{2}}\right)=\left(f, \psi_{i j}^{(1)}\right)+O\left(h^{6}\right)\left\|u_{h}\right\|_{2, \infty, e_{i j}},
$$

which, together with the following relation obtained by a simple calculation,

$$
\left(f, \psi_{i j}^{(1)}\right)=-\frac{h_{i}^{3} k_{j}}{12} f(p)+O\left(h^{4+k}\right)\|f\|_{k, \infty, e_{i j}}, \quad k=0,1,2,
$$

yields

$$
\begin{aligned}
\frac{\partial^{2} u_{h}(p)}{\partial x^{2}}= & -\frac{1}{A_{1}(p)}\left(\frac{\partial A_{1}(p)}{\partial x} \frac{\partial u_{h}(p)}{\partial x}-B(p) u_{h}(p)+f(p)\right) \\
& +O\left(h^{2}\right)\left\|u_{h}\right\|_{2, \infty, e_{i j}}+O\left(h^{k}\right)\|f\|_{k, \infty, e_{i j}} .
\end{aligned}
$$

A similar relation holds also for $\frac{\partial^{2} u_{h}(p)}{\partial y^{2}}$. This completes the proof. Q.E.D. 
Corollary 1 The following stability estimate holds true:

$$
\left\|u_{h}\right\|_{2, \infty, h} \leq c\|u\|_{2, \infty, \Omega}
$$

Proof. By standard interpolation estimates and Lemma 4, we get

$$
\begin{aligned}
\left\|u_{h}-I_{h} u_{h}\right\|_{2, \infty, h} & \leq c\left(\left\|\frac{\partial^{2} u_{h}}{\partial x^{2}}\right\|_{0, \infty, h}+\left\|\frac{\partial^{2} u_{h}}{\partial y^{2}}\right\|_{0, \infty, h}\right) \\
& \leq c\|f\|_{0, \infty, \Omega}+c\left\|u_{h}\right\|_{1, \infty, h}+c h^{2}\left\|u_{h}\right\|_{2, \infty, h} \\
& \leq c\|f\|_{0, \infty, \Omega}+c\left\|I_{h} u_{h}\right\|_{1, \infty, \Omega}+c h\left\|u_{h}\right\|_{2, \infty, h} .
\end{aligned}
$$

Moreover, by Lemma 3 and the $W^{2, \infty}$-stability for conforming elements (see [11], [17]), it follows that

$$
\begin{aligned}
\left\|I_{h} u_{h}\right\|_{2, \infty, h} & \leq c\left\|I_{h} u_{h}-\bar{u}_{h}\right\|_{2, \infty, h}+c\left\|\bar{u}_{h}\right\|_{2, \infty, h} \\
& \leq c h\left\|u_{h}\right\|_{2, \infty, h}+c\|u\|_{2, \infty, \Omega} .
\end{aligned}
$$

Therefore, we conclude that

$$
\begin{aligned}
\left\|u_{h}\right\|_{2, \infty, h} & \leq\left\|I_{h} u_{h}-u_{h}\right\|_{2, \infty, h}+\left\|I_{h} u_{h}\right\|_{2, \infty, h} \\
& \leq c h\left\|u_{h}\right\|_{2, \infty, h}+c\|u\|_{2, \infty, \Omega}
\end{aligned}
$$

which, for sufficiently small $h$, yields the asserted inequality. Q.E.D.

\section{The proofs of the theorems}

In this section we give all proofs of the three theorems stated in section 2 .

Proof of Theorem 1. Fix $e_{i j} \in T_{h}$ and $z \in Z_{h} \cap e_{i j}$. By Lemma 4 we find that

$$
L u_{h}(z)+\left(B u_{h}\right)(z)=2 f(z)+O\left(h^{2}\right)\left\|u_{h}\right\|_{2, \infty, e_{i j}}+O(h)\|f\|_{1, \infty, e_{i j}} .
$$

Replacing $z$ by an arbitary point on $e_{i j}$ in (10) we obtain

$$
L u_{h}+B u_{h}=2 f+O(h)\left(h\left\|u_{h}\right\|_{2, \infty, e_{i j}}+\|f\|_{1, \infty, e_{i j}}\right),
$$

which implies that

$$
B u-f=L\left(u-u_{h}\right)+O(h)\left(h\left\|u_{h}\right\|_{2, \infty, h}+\|f\|_{1, \infty, \Omega}\right) .
$$


By the inverse estimate and the following approximation property

$$
\inf _{\chi \in V_{h}}\|\varphi-\chi\|_{k, p, \Omega} \leq c h^{3-k}\|\varphi\|_{3, p, \Omega}, \quad \text { for all } \quad \varphi \in W^{3, p}(\Omega) \cap W_{0}^{1, p}(\Omega),
$$

where $k=0,1,2$, we have, by (11), Lemma 4 and Corollary 1, that

$$
\begin{aligned}
\|B u-f\|_{0, p, \Omega} & \leq c\left\|L\left(u-u_{h}\right)\right\|_{0, p, h}+c h\left\|u_{h}\right\|_{1, \infty, h}+c h\|f\|_{1, \infty, \Omega} \\
& \leq c h^{k-2}\left\|u-u_{h}\right\|_{k, p, h}+c h\|u\|_{3, \infty, \Omega} .
\end{aligned}
$$

This proves the theorem, as $h$ becomes small enough, provided that $u \not \equiv 0$ implying that

$$
\|B u-f\|_{0, p, \Omega}>0
$$

Q.E.D.

Proof of Theorem 2. Let $p \in Z_{h}$ be fixed. Suppose $p \in e_{i j}$ for some $e_{i j} \in T_{h}$. A simple calculation shows that

$$
\nabla\left(I_{h} u_{h}-u_{h}\right)(p)=0 .
$$

Now applying the superconvergence estimate for the conforming bilinear finite element method (see [18] and [7])

$$
\left|\nabla\left(u-\bar{u}_{h}\right)(p)\right| \leq c h^{2} \ln \frac{1}{h}\|u\|_{3, \infty, \Omega},
$$

and using Lemma 3 and Corollary 1 we find that

$$
\begin{aligned}
\left|\nabla\left(u-u_{h}\right)(p)\right| & \leq c\left|\nabla\left(u-\bar{u}_{h}\right)(p)\right|+\left|\nabla\left(\bar{u}_{h}-I_{h} u_{h}\right)(p)\right| \\
& \leq c h^{2} \ln \frac{1}{h}\|u\|_{3, \infty, \Omega} .
\end{aligned}
$$

This completes the proof. Q.E.D.

To prove Theorem 3 we need the following result, which provides an asymptotic expansion for the second derivative of $u_{h}$ and plays an important role in deriving all estimates in Theorem 3.

Lemma 5 Let $u_{h}$ be the solution of problem (2). If the solution $u$ of problem (1) belongs to $W^{4, \infty}(\Omega)$, then, for $p \in Z_{h}$, there holds

$$
\partial_{s}^{2} u_{h}(p)=F_{s}(p)+O\left(h^{2} \ln \frac{1}{h}\right)\|u\|_{4, \infty, \Omega},
$$

where $F_{s}(s=1,2)$ are sufficiently smooth functions on $\Omega$ independent of $h$. 
Proof. Let $p \in Z_{h} \cap e_{i j}$ for some $e_{i j} \in T_{h}$. By the expansion of $u_{h}$ with respect to the basis functions of $\bar{V}_{h}$, and by Lemma 2, we have

$$
u_{h}=I_{h} u_{h}+O\left(h^{2}\right)\|u\|_{2, \infty, \Omega} .
$$

Hence, by the $L^{\infty}$ estimate for the bilinear finite element approximation, and by Lemma 3, we have

$$
\left|u(p)-u_{h}(p)\right| \leq c h^{2} \ln \frac{1}{h}\|u\|_{2, \infty, \Omega} .
$$

Now taking $k=2$ in Lemma 4 and using Theorem 2, we get

$$
\begin{aligned}
\frac{\partial^{2} u_{h}(p)}{\partial x^{2}}= & -\frac{1}{A_{1}(p)}\left(\frac{\partial A_{1}(p)}{\partial x} \frac{\partial u(p)}{\partial x}-B(p) u(p)+f(p)\right) \\
& +O\left(h^{2} \ln \frac{1}{h}\right)\|u\|_{4, \infty, \Omega} .
\end{aligned}
$$

This proves the lemma for $s=1$, with

$$
F_{1}=-\frac{1}{A_{1}}\left(\frac{\partial A_{1}}{\partial x} \frac{\partial u}{\partial x}-B u+f\right) .
$$

A similar relation holds true for $s=2$. Q.E.D.

Now we are in the position to prove Theorem 3.

Proof of Theorem 3. It suffices to show, for $q \in N_{h}$, that

$$
u(q)-u_{h}(q)=I_{h}\left(u-u_{h}\right)(q)=\sum_{e_{i j} \in T_{h}}\left(h_{i}^{2} \phi_{1}+k_{j}^{2} \phi_{2}\right)+\bar{\gamma}_{h},
$$

where $\phi_{1}, \phi_{2}$ are two functions independent of $h$, and where the remainder $\bar{\gamma}_{h}$ admits the same estimates as those for $\gamma_{h}$ in Theorem 3 .

Let $g_{h}^{q} \in \bar{V}_{h}$ denote the discrete Green's function defined by,

$$
a\left(\varphi_{h}, g_{h}^{q}\right)=\varphi_{h}(q), \quad \text { for } \quad \varphi_{h} \in \bar{V}_{h},
$$

and let $g^{q}$ denote the Green's function at $q$. Then, as it is well-known that (see, for example, [6] and [18]),

$$
\begin{gathered}
\left(h \ln \frac{1}{h}\right)^{-1}\left\|g^{q}-g_{h}^{q}\right\|_{0,1, \Omega}+\left\|g^{q}-g_{h}^{q}\right\|_{1,1, h}+h\left\|g_{h}^{q}\right\|_{2,1, h} \\
+h \ln \frac{1}{h}\left\|g_{h}^{q}\right\|_{0,2, \Omega} \leq c h \ln \frac{1}{h}
\end{gathered}
$$


Furthermore, for $q \in \Omega_{0}$ and $\varphi \in W^{1,1+\epsilon}\left(\Omega_{0}\right) \cap L^{2}(\Omega)$, one has, [6], [18],

$$
\left|\left(g^{q}-g_{h}^{q}, \varphi\right)\right| \leq c h^{2} \ln \frac{1}{h}\|\varphi\|_{1,2+\epsilon, \Omega_{0}}+c h^{2}\|\varphi\|_{0,2, \Omega},
$$

where $\Omega_{0} \subset \subset \Omega_{1} \subset \Omega$. Now we have

$$
=\sum_{e_{i j} \in T_{h}} \int_{e_{i j}}\left\{A_{1} \frac{\partial\left(I_{h} u_{h}-u_{h}\right)}{\partial x} \frac{\partial g_{h}^{q}}{\partial x}+A_{2} \frac{\partial\left(I_{h} u_{h}-u_{h}\right)}{\partial y} \frac{\partial g_{h}^{q}}{\partial y}+B\left(I_{h} u_{h}-u_{h}\right) g_{h}^{q}\right\} d x d y,
$$

where $\bar{u}_{h}$ is the bilinear finite element approximation of $u$. By Lemma 2, Lemma 5 , Corollary 1 and (15), for $q \in \bar{\Omega}$, we have

$$
\begin{aligned}
\sum_{e_{i j} \in T_{h}} \int_{e_{i j}} B\left(I_{h} u_{h}-u_{h}\right) g_{h}^{q} d x d y= & \frac{1}{12} \sum_{e_{i j} \in T_{h}} \int_{e_{i j}} B g_{h}^{q}\left(h_{i}^{2} \frac{\partial^{2} u_{h}}{\partial x^{2}}+k_{j}^{2} \frac{\partial^{2} u_{h}}{\partial y^{2}}\right) d x d y \\
& +O\left(h^{4} \ln \frac{1}{h}\right)\left\|u_{h}\right\|_{2, \infty, h} \\
= & \frac{1}{12} \sum_{e_{i j} \in T_{h}} \int_{e_{i j}} B g_{h}^{q}\left(h_{i}^{2} F_{1}(p)+k_{j}^{2} F_{2}(p)\right) d x d y \\
& +O\left(h^{4} \ln \frac{1}{h}\right)\|u\|_{3, \infty, \Omega} \\
= & \frac{1}{12} \sum_{e_{i j} \in T_{h}} \int_{e_{i j}} B g^{q}\left(h_{i}^{2} F_{1}+k_{j}^{2} F_{2}\right) d x d y+R_{1},
\end{aligned}
$$

where the remainder satisfies

$$
\left|R_{1}\right| \leq c h^{4} \begin{cases}\left(\ln \frac{1}{h}\right)^{2}\|u\|_{4, \infty, \Omega}, & \text { if } \quad q \in \bar{\Omega}, \\ \ln \frac{1}{h}\left(\|f\|_{1,2+\epsilon, \Omega_{0}}+\|u\|_{3, \infty, \Omega}\right), & \text { if } \quad q \in \Omega_{0} \text { and } T_{h} \text { is u.u., }\end{cases}
$$

where u.u. means unidirectionally uniform. On the other hand, by Lemma 2, Corollary 1 and (13) we get

$$
\begin{aligned}
\sum_{e_{i j} \in T_{h}} \int_{e_{i j}} A_{1} \frac{\partial\left(I_{h} u_{h}-u_{h}\right)}{\partial x} \frac{\partial g_{h}^{q}}{\partial x} d x d y= & -\frac{1}{12} \sum_{e_{i j} \in T_{h}} \int_{e_{i j}} h_{i}^{2} \frac{\partial A_{1}}{\partial x} \frac{\partial g_{h}^{q}}{\partial x} \frac{\partial^{2} u_{h}}{\partial x^{2}} d x d y \\
& +O\left(h^{4} \ln \frac{1}{h}\right)\left\|u_{h}\right\|_{2, \infty, h} \\
= & -\frac{1}{12} \sum_{e_{i j} \in T_{h}} \int_{e_{i j}} h_{i}^{2} \frac{\partial A_{1}}{\partial x} \frac{\partial g_{h}^{q}}{\partial x} F_{1} d x d y \\
& +O\left(h^{4} \ln \frac{1}{h}\right)\|u\|_{4, \infty, \Omega} .
\end{aligned}
$$


This, together with (13), leads to the following relation

$$
\begin{aligned}
\sum_{e_{i j} \in T_{h}} \int_{e_{i j}} A_{1} \frac{\partial\left(I_{h} u_{h}-u_{h}\right)}{\partial x} \frac{\partial g_{h}^{q}}{\partial x} d x d y= & -\frac{1}{12} \sum_{e_{i j} \in T_{h}} h_{i}^{2} \int_{e_{i j}} \frac{\partial A_{1}}{\partial x} \frac{\partial g^{q}}{\partial x} F_{1} d x d y \\
& +O\left(h^{3} \ln \frac{1}{h}\right)\|u\|_{3, \infty, \Omega} .
\end{aligned}
$$

If $T_{h}$ is unidirectionally uniform, i.e., $h_{i}=h_{1}$, then by integrating by parts, we have

$$
\sum_{e_{i j} \in T_{h}} \int_{e_{i j}} A_{1} \frac{\partial\left(I_{h} u_{h}-u_{h}\right)}{\partial x} \frac{\partial g_{h}^{q}}{\partial x} d x d y=\frac{h_{i}^{2}}{12} \int_{\Omega} \frac{\partial}{\partial x}\left(\frac{\partial A_{1}}{\partial x} F_{1}\right) g^{q} d x d y+R_{2},
$$

where $R_{2}$ can be estimated by the same bound as for $R_{1}$ (cf. (18)). A similar expansion holds for the other term containing $A_{2}$. In view of (15), we see that

$$
\left(I_{h} u_{h}-\bar{u}_{h}\right)(q)=\sum_{e_{i j} \in T_{h}} \int_{e_{i j}}\left(h_{i}^{2} E_{1}+k_{j}^{2} E_{2}\right) d x d y+R
$$

where $R$ admits the same bound as $\gamma_{h}$ in Theorem 3 , and $E_{1}, E_{2}$ are functions independent of $h$. Finally we apply an error expansion for the conforming bilinear element (see [1], [3], [10] and [18]) to get

$$
\left(I_{h} u-\bar{u}_{h}\right)(q)=\sum_{e_{i j} \in T_{h}} \int_{e_{i j}}\left(h_{i}^{2} L_{1}+k_{j}^{2} L_{2}\right) d x d y+O\left(h^{4-\sigma} \ln \frac{1}{h}\right)\|u\|_{4-\sigma, \infty, \Omega}
$$

where $\sigma=0$ or 1 , corresponding to a quasi-uniform $T_{h}$ or a unidirectionally uniform $T_{h}$, respectively, and where $L_{1}, L_{2}$ are again two functions independent of $h$. Combining (17) and (18) we get (12). This completes the proof. Q.E.D.

\section{On the error behavior in negative norms}

In this final section we consider the error behavior in negative Sobolev norms. To show that there is generally no improvement in the order by going to weaker norms, we construct a particular solution $u$ satisfying (5). For simplicity and without loss of generality, we consider a model problem with $L=-\Delta$, and assume that the mesh $T_{h}$ is uniform with $h=h_{i}=k_{j}$ (for all $i$ and $j$ ). Let us first note that, for any $\varphi \in C_{0}^{\infty}(\Omega)$, there holds

$$
\left(u-\bar{u}_{h}, \varphi\right)=-\frac{h^{2}}{6}\left(\frac{\partial^{2} \Phi}{\partial y^{2}}, \frac{\partial^{2} u}{\partial x^{2}}\right)+\frac{h^{2}}{12}(f, \varphi)+O\left(h^{4}\right)\left\|\nabla^{2} \varphi\right\|,
$$


where $\Phi \in W_{0}^{1,2}(\Omega) \cap W^{2,2}(\Omega)$ satisfies $-\Delta \Phi=\varphi$ in $\Omega$. The expansion (21) can be easily obtained by using the relation

$$
\left(u-\bar{u}_{h}, \varphi\right)=\left(\nabla \bar{u}_{h}, \nabla\left(I_{h} \Phi-\Phi\right)\right)+\left(f, \Phi-I_{h} \Phi\right)
$$

and the well-known error expansions for the interpolation operator, see, e.g., [1], [3], or [10]. Secondly, according to Lemma 5, we have $-\left(\Delta u_{h}, \varphi\right)=2(f, \varphi)+O\left(h^{2}\right)\|\varphi\|$ for all $\varphi \in C_{0}^{\infty}(\Omega)$. Thus, by the known error expansions for the interpolation, we obtain

$$
\left(I_{h} u_{h}-u_{h}, \varphi\right)=-\frac{h^{2}}{6}(f, \varphi)+O\left(h^{4}\right)\left\|\nabla^{2} \varphi\right\| .
$$

Furthermore, since

$$
\begin{aligned}
\left\|\nabla\left(I_{h} u_{h}-\bar{u}_{h}\right)\right\|^{2} & =\left(\nabla\left(I_{h} u_{h}-\bar{u}_{h}\right), \nabla\left(I_{h} u_{h}-\bar{u}_{h}\right)\right) \\
& =\left(\nabla\left(I_{h} u_{h}-u_{h}, \nabla\left(I_{h} u_{h}-\bar{u}_{h}\right)\right)+\left(\nabla\left(u_{h}-\bar{u}_{h}\right), \nabla\left(I_{h} u_{h}-\bar{u}_{h}\right)\right)\right. \\
& =0+0=0,
\end{aligned}
$$

we have

$$
I_{h} u_{h}=\bar{u}_{h}
$$

Now, the combination of (21), (22) and (23) leads to

$$
\begin{aligned}
\left(u-u_{h}, \varphi\right) & =\left(u-\bar{u}_{h}, \varphi\right)+\left(I_{h} u_{u}-u_{h}, \varphi\right) \\
& =-\frac{h^{2}}{6}\left(\frac{\partial^{2} \Phi}{\partial y^{2}}, \frac{\partial^{2} u}{\partial x^{2}}\right)-\frac{h^{2}}{12}(f, \varphi)+O\left(h^{4}\right)\left\|\nabla^{2} \varphi\right\| .
\end{aligned}
$$

Hence, if $\Phi \in C_{0}^{\infty}(\Omega)$, there holds

$$
\left(u-u_{h}, \varphi\right)=-\frac{h^{2}}{12}\left\{\left(\Delta^{2} u+2 \frac{\partial^{4} u}{\partial x^{2} \partial y^{2}}, \Phi\right)\right\}+O\left(h^{4}\right)\left\|\nabla^{2} \varphi\right\| .
$$

Let $w \in W_{0}^{1,2}(\Omega) \cap W^{2,2}(\Omega)$ satisfy $-\Delta w=\Delta^{2} u+2 \frac{\partial^{4} u}{\partial x^{2} \partial y^{2}}$ in $\Omega$, then

$$
\left(u-u_{h}+\frac{h^{2}}{12} w, \varphi\right)=O\left(h^{4}\right)\left\|\nabla^{2} \varphi\right\|
$$

The identity (25) shows that the estimate (5) holds true if $\Delta^{2} u+2 \frac{\partial^{4} u}{\partial x^{2} \partial y^{2}} \neq 0$, implying that $w \neq 0$. 
Acknowledgements. The authors thank Prof. Z. C. Shi for drawing their attention to the open problem of higher order error estimates for the Wilson nonconforming finite element. They also like to thank Prof. Dr. R. Rannacher and Prof. Dr. H. Blum for their many helpful discussions and suggestions.

Institut Für Angewandte Mathematik

Universität Heidelberg

Im Neuenheimer Feld 293

W-6900 Heidelberg, Germany

email: bx1@vm.urz.uni-heidelberg.de

School of Mathematics

University of Minnesota

127 Vincent Hall, 206 Church Street S.E.

Minneapolis, MN 55455, U.S.A.

email: bli@math.umn.edu

\section{References}

[1] H. Blum, Asymptotic error expansion and defect correction in the finite element method, Preprint, Habilitationsscrift, Univ. Heidelberg, July, 1990.

[2] H. Blum, Q. Lin, R. Rannacher, Asymptotic error expansion and Richardson extrapolation for linear finite element, Numer. Math., 49, 11-37 (1986).

[3] H. Chen, A rectangular finite element analysis, Natur. Sci. J. Xiangtan Univ., 11, 1-11(1989)(in Chinese).

[4] H. Chen, Q. Lin, Finite element approximation using a graded mesh on domains with reentrant corners, Syst. Sci. \& Math. scis., 5(2), 127-140(1992).

[5] P. G. Ciarlet, The Finite Element Method for Elliptic Problems, North-Holland, 1978.

[6] J. Frehse, R. Rannacher, $L^{1}$ - Fehlerabschätzung für diskrete Grundlösunger in der Methode der finite Elemente, Bonn. Math. Schr., 89, 92-114(1979).

[7] M. Krizek, P. Neittaanmäki, On Superconvergence techniques, Acta Appl. Math., 9, 175-198 (1987). 
[8] P. Lesaint, M. Zlamal, Convergence of the nonconforming Wilson element for arbitary quadrilateral meshes, Numer. Math., 36, 33-82 (1980).

[9] B. Li, On superconvergence for higher-order triangular finite elements, Chinese J. Numer. Math. Appl., 12(1), 75-79 (1990).

[10] Q. Lin, Fourth order eingenvalue approximation by extrapolation on domains with reentrant corner, Numer. Math., 58, 631-640 (1991).

[11] R. Rannacher, R. Scott, Some optimal error estimates for piecewise linear finite element approximations, Math. Comp., 38, 437-445 (1982).

[12] S. M. Shen, $L^{\infty}$-convergence of nonconforming finite element approximations, Math. Numer. Sinica, 8(3), 225-230 (1986) (in Chinese).

[13] Z. C. Shi, A convergence condition for the quadrilateral Wilson element, Numer. Math., 44, 349-361 (1984).

[14] Z. C. Shi, A remark on the optimal order of convergence of Wilson nonconforming element, Math. Numer. Sinica, 8(2), 159-163 (1986) (in Chinese).

[15] G. Strang, G. J. Fix, An Analysis of the Finite Element Method, Prentice-Hall, Englewood Cliffs, New Jersey, 1973.

[16] H. Wang, On superconvergence of Wilson element, J. Shandong Univ., 21(4), (1986) (in Chinese).

[17] O. C. Zienkiewicz, The Finite Element Method, 3rd ed, McGraw-Hill, New York, 1977.

[18] Q. D. Zhu, Q. Lin, Superconvergence Theory of the Finite Element Methods, Hunan Science Press, 1990 (in Chinese). 